\title{
Assurer la sécurité alimentaire dans un contexte de changement climatique : un défi collectif
}

\author{
Marion GUILLOU \\ Institut National de la Recherche \\ Agronomique (INRA), \\ 147 rue de I'Université - 75338 Paris \\ Cedex 07 ; \\ Commission internationale sur \\ I'Agriculture durable et le Changement \\ climatique; \\ Agreenium \\ $<$ marion.guillou@agrenium.org >
}

Article reçu le 12 juillet 2012

Accepté le 30 juillet 2012

\begin{abstract}
Achieve food security in the face of climate change: a collective issue There is an urgent need to renew our agricultural model: 1 billion people still suffer from starvation nowadays, we are running short with fossile resources and climate change is already transforming agriculture throughout the World. Food security is at stake for the decades to come and most of the international decision makers are becoming aware of it, as illustrated by a series of conferences and summits which include: the G20 summit on agriculture in Paris (June 2011) and the Earth Summit which took place in Rio on last June.

However, while preparing for those important meetings decision-makers were in need for an accurate and comprehensive information on the latest scientific results. This is the reason why the international commission on sustainable agriculture and climate change was set up in 2011.

Made of 13 eminent scientists, this international commission was in charge of identifying which policy changes and actions are needed to help the world achieve food security in the face of climate change. After 15 months of working, the commission issued its final report in March 2012 and this article is aimed at introducing the main conclusions of this report.
\end{abstract}

Key words: climate change, food security, environment, agriculture, resources, commission, report, summit, decision-makers

à l'épuisement des ressources naturelles, à l'exode rural, aux migrations transfrontalières, mais aussi à l'instabilité politique et économique.

Dans les années passées, la révolution verte a permis de faire mieux que suivre l'évolution démographique. Mais le modèle agricole et alimentaire doit être renouvelé. En effet, I'agriculture contribue de manière significative aux émissions de gaz à effet de serre, à cause notamment de certaines pratiques (défrichage, mauvaise utilisation des engrais et des résidus organiques, élevage hors sol. . .). De plus, et à mesure que la demande mondiale de nourriture, de fourrage et de cultures bioénergétiques augmente, de nombreux systèmes agricoles appauvrissent les sols, la biodiversité et les ressources en eau. Ainsi, dans de nombreuses régions, les écarts entre les rendements potentiels et réels des cultures sont énormes. Chaque année, on estime que 12 millions d'hectares de terres agricoles, susceptibles de produire potentiellement 20 millions de tonnes de céréales, sont perdus en raison de la dégradation des sols; ils viennent s'ajouter aux milliards d'hectares qui sont déjà dégradés (United Nations Convention to Combat Desertification, 2011 ; Bai et al., 2008). Enfin, on estime qu'un tiers de la nourriture produite pour la consommation humaine est perdu ou gaspillé dans le système alimentaire mondial (United Nations Convention to Combat Desertification, 2011).

À ce tableau, il convient d'ajouter également un autre facteur important, le changement climatique, qui devrait se poursuivre dans les décennies à venir. Ce processus est déjà perceptible par les événements climatiques (températures élevées, sécheresses, inondations...) d'une grande souffrance, cette insécurite alimentaire contribue à la dégradation et 
dont la fréquence et la gravité se sont accrues au cours des dernières années. Tant sur le plan social qu'économique ou écologique, ces événements extrêmes touchent directement de nombreuses populations à travers le monde. De plus, les températures moyennes à la surface du globe risquent de connaître une augmentation si les émissions de gaz à effet de serre ne changent pas de manière drastique. Or, le changement climatique aura un impact global indésirable sur la production agricole dans les décennies à venir : dans de nombreuses régions, les seuils critiques seront approchés, voire dépassés, et c'est dans les zones qui souffrent actuellement de l'insécurité alimentaire que le changement climatique emportera les conséquences les plus néfastes. Pour réduire les effets du changement climatique sur I'agriculture et sur I'alimentation, il est d'ores et déjà impératif de renforcer de manière significative la capacité des systèmes agricoles à s'adapter tant aux tendances climatiques de long terme qu'à l'augmentation de la variabilité.

Les décideurs publics commencent à prendre la mesure de la situation: la sécurité alimentaire a été au centre des débats lors de la réunion que le G20, sous présidence française, a consacrée à l'agriculture en juin 2011 et elle a également été abordée lors du Sommet "Rio + 20 » en juin 2012. Dans ce contexte, il était important qu'ils puissent disposer d'une information scientifique fiable et à jour : c'est dans cette perspective que la Commission internationale sur l'Agriculture durable et le Changement climatique avait été mise en place au début de l'année 2011.

Cette Commission s'inscrit dans le cadre d'un ambitieux programme que le Groupe consultatif sur la recherche agricole internationale (acronyme en Anglais, CGIAR) a lancé en 2009 autour de l'agriculture, de I'alimentation et du changement climatique ${ }^{1}$. Composée de 13 experts venus de pays du Nord comme de pays du Sud, elle est présidée par Sir John Beddington, Chief Scientific Adviser du gouvernement britannique. Tout au long de l'année 2011, cette commission internationale a passé en revue des dizaines de résultats et de

\footnotetext{
${ }^{1}$ CGIAR Research Program on Climate Change, Agriculture and Food Security (CCAFS).
}

Tableau 1. Paramètres retenus par la Commission

\begin{tabular}{|c|c|}
\hline Population mondiale (2011) ${ }^{1}$ & 7 milliards \\
\hline Personnes souffrant de malnutrition $(2010)^{2}$ & 0,9 milliard \\
\hline Personnes de plus de 20 ans en surpoids $(2008)^{3}$ & 1,5 milliard \\
\hline Personnes vivant avec moins de 1,25 \$US par jour $(2005)^{4}$ & 1,4 milliard \\
\hline Personnes vivant dans les zones arides $(2007)^{5}$ & 2 milliards \\
\hline Personnes dépendantes de la détérioration des sols ${ }^{6}$ & 1,5 milliard \\
\hline $\begin{array}{l}\text { Pertes dues aux évènements climatiques } \\
\text { (températures extrêmes, sécheresse, feu de forêt) }(2010)^{7}\end{array}$ & 7,5 milliards $\$ U S$ \\
\hline Surfaces des terres agricoles $(2009)^{8}$ & 4,9 milliards d'hectares \\
\hline $\begin{array}{l}\text { Zones de cultures, pâturages et surfaces herbagères } \\
\text { consacrées à l'élevage des animaux }{ }^{9}\end{array}$ & 3,7 milliards d'hectares \\
\hline $\begin{array}{l}\text { Croissance annuelle de la production } \\
\text { agricole mondiale }(1997-2007)^{10}\end{array}$ & $2,2 \%$ \\
\hline $\begin{array}{l}\text { Nourriture produite pour la consommation } \\
\text { humaine perdue et gaspillée chaque année } \\
\end{array}$ & 1,3 milliard de tonnes \\
\hline
\end{tabular}

1. http://www.un.org/en/development/desa/news/population/world-to-welcome-seven-billionth-citizen.html.

2. FAO (2010).

3. World Health Organization (2011).

4. Banque Mondiale : Données sur la Pauvreté et l'Equité.

http://povertydata.worldbank.org/poverty/home/

5. United Nations Convention to Combat Desertification (2011).

6. Ibid.

7. Munich RE NatCatSERVICE (2010).

8. Foley et al. (2011).

9. Ibid.

10. Bruinsma (2009).

11. Gustavsson et al., 2011.

travaux de synthèse scientifiques : elle a édicté une série de recommandations en décembre 2011 à l'attention des décideurs publics ${ }^{2}$, puis un rapport complet, rendu public en mars $2012^{3}$ (tableau 1).

Les recommandations comme le rapport de la Commission I'affirment clairement: pour nourrir une population mondiale en pleine croissance, il faudra tout d'abord accroître la production agricole. Remplir ce premier impératif suppose de favoriser la résilience de la

${ }^{2}$ CGIAR, Research Program on Climate Change, Agriculture and Food Security (CCAFS), Commission on Sustainable Agriculture and Climate Change, Achieving Food Security in the Face of Climate Change, Summary for Policy Makers, déc. 2011. http://ccafs.cgiar. org/sites/default/files/assets/docs/climate_ food_commission-spm-nov2011.pdf

${ }^{3}$ id., Achieving Food Security in the Face of Climate Change, Final Report, mars 2012. http://ccafs.cgiar.org/sites/default/files/ assets/docs/climate_food_commission-finalmar2012.pdf production (cultures, élevage) face aux chocs climatiques, mais aussi de stopper la dégradation des sols tout en favorisant le stockage du carbone.

Pour autant, la Commission sur I'Agriculture durable et le Changement climatique estime également que l'augmentation de la production doit prendre en compte les impératifs du développement durable. Investir davantage, accroître l'innovation et cibler prioritairement les populations les plus vulnérables: toutes ces actions doivent contribuer à construire un système alimentaire mondial qui garantisse mieux la sécurité alimentaire.

En résumé, dans ses recommandations et dans son rapport final, la Commission sur I'Agriculture durable et le Changement climatique prône une intensification de la production alimentaire combinée avec une action concertée pour limiter l'accélération du changement climatique. Toutefois, la diversité tant écologique qu'humaine qui caractérise notre planète interdit d'imposer des solutions uniques: il conviendra avant tout de 
s'appuyer sur les pratiques locales et sur les expériences couronnées de succès. Enfin, dans son rapport final, la Commission émet à l'attention des décideurs sept recommandations concrètes : sur la base de ces recommandations, la Commission voudrait inviter à une révision complète du système alimentaire mondial; ses membres soulignent surtout la nécessité de mettre en œuvre très rapidement ces mesures.

\section{Première recommandation : intégrer la sécurité alimentaire et I'agriculture durable dans les politiques internationales et nationales}

Dans une première recommandation, la Commission sur l'Agriculture durable et le Changement climatique appelle à intégrer la sécurité alimentaire et l'agriculture durable dans les politiques internationales et nationales.

En ce qui concerne l'agriculture plus particulièrement, il convient tout d'abord d'établir un programme de travail qui porte aussi bien sur l'atténuation du changement climatique que sur l'adaptation à ce dernier. Ce programme de travail devra suivre les principes et les dispositions de la Convention Cadre des Nations Unies sur le Changement Climatique (CCNUCC). II constituera une première étape vers l'inclusion de l'agriculture dans les politiques internationales relatives à la lutte contre le changement climatique.

Pour la Commission, l'agriculture durable et respectueuse de l'environnement devait impérativement constituer un élément central de l'initiative Croissance verte $^{4}$ et du Sommet de la Terre Rio $+20^{5}$.

Plus concrètement, il est important de financer des actions pour l'évolution des systèmes de production agricole dans

\footnotetext{
${ }^{4}$ Green Growth (Croissance verte) est une politique qui met l'accent sur la progression économique durable en termes écologiques afin d'encourager un développement inclusif sur le plan social et à faible empreinte carbone. www.greengrowth.org

${ }^{5}$ Rio+20, Conférence des Nations Unies sur le Développement Durable, Rio de Janeiro, Brésil 4-6 juin 2012.
}

l'objectif d'atteindre une meilleure résilience à la variabilité et aux chocs météorologiques, tout en contribuant de manière significative à atténuer le changement climatique. Parmi ces actions, on peut citer : le soutien aux évaluations nationales sur le changement climatique, les développements des stratégies d'atténuation et d'adaptation...

La Commission appelle également à développer des plateformes de dialogue, que ce soit à l'échelon international, national et régional. L'objectif : parvenir à des actions politiques coordonnées dans les domaines du changement climatique, de l'agriculture, de la réponse face à la crise et à la sécurité alimentaire aux différents niveaux. La mise en place $d^{\prime}$ 'un tel dialogue suppose ainsi d'encourager les coalitions nationales pour la sécurité alimentaire.

Le Brésil offre un exemple de mise en place d'une politique volontariste. Dans ce pays, la déforestation et les changements dans l'usage des terres expliquent plus de $70 \%$ des émissions de gaz à effet de serre (World Bank, 2010a). Pourtant, l'exemple brésilien montre que les gouvernements nationaux peuvent stimuler la production alimentaire durable par des politiques coordonnées. En plus de ses succès économiques et sociaux - une réduction de la pauvreté à $7 \%$ de la population en 2010 , contre $20 \%$ en 2004 - le Brésil a déployé des politiques et de programmes complémentaires pour réduire l'impact de l'agriculture sur I'environnement. Cinquième plus gros émetteur de GES au monde, le Brésil va pourtant tenir $80 \%$ des engagements qu'il avait pris dans les secteurs agricole et forestier au titre de l'Accord de Copenhague. Or, ces deux secteurs génèrent plus de $70 \%$ de ses émissions. Entré en vigueur en 2008, le plan national brésilien contre le changement climatique est crédité de succès dans le passage à une agriculture à faibles émissions de GES. Les lois de zonage agro-écologique sur la canne à sucre et I'huile de palme permettent de limiter les effets négatifs liés à la concurrence sur I'usage des terres. Parallèlement, ces lois répondent à plusieurs objectifs dans les secteurs de l'agriculture, de la forêt et de l'énergie. Le Plan national, le Plan pour une Amazonie durable et le Plan sur les ressources nationales en eau interdisent ainsi la culture de canne à sucre dans les zones protégées, l'Amazonie et le Pantanal.
Deuxième

recommandation : augmenter de manière significative le niveau des investissements mondiaux dans I'agriculture durable et les systèmes alimentaires

Dans une deuxième recommandation, la Commission exhorte à augmenter de manière significative le niveau des investissements mondiaux dans l'agriculture durable et les systèmes alimentaires au cours de la prochaine décennie.

Lors du sommet du G8 tenu à l'Aquila (Italie) en juillet 2009, des engagements ont été pris et des programmes ont été lancés par les dirigeants internationaux : ces engagements, y compris ceux de long terme, et ces programmes doivent maintenant être pleinement mis en œuvre, que ce soit pour l'assistance financière et technique dans la production alimentaire ou pour les petits exploitants agricoles.

Dans le même ordre d'idées, les mécanismes comme le Fonds "Fast Start " (émanation de la Conventioncadre des Nations-Unies sur les Changements Climatiques), mais aussi les grandes banques de développement doivent désormais mettre l'accent sur les programmes d'agriculture durable qui apportent la sécurité alimentaire, des conditions de vie meilleures, une résilience au changement climatique et simultanément des avantages écologiques. Quant à ces programmes euxmêmes, ils doivent mettre en avant les impératifs d'amélioration des infrastructures et de réhabilitation des terres.

Au niveau national, il importe d'augmenter les budgets de recherche et de développement pour des agricultures durables, afin de bien refléter l'importance de celles-ci dans la croissance économique, la baisse de la pauvreté et le respect des impératifs de durabilité. Les programmes nationaux de recherche devront également mettre l'accent sur les problèmes-clés de sécurité alimentaire, par exemple le développement des cultures alimentaires autres que les céréales ou la réduction des pertes post-récoltes.

Plus globalement, la Commission estime qu'il convient d'accroître les 
connaissances sur les bonnes pratiques et l'accès à l'innovation, en soutenant non seulement le renouvellement des outils de diffusion de la connaissance, mais aussi le transfert de technologies entre les communautés qui partagent les mêmes bonnes pratiques (par exemple échanges Nord-Sud, Sud-Nord, et d'agriculteur à agriculteur). Ici une attention toute particulière devra être consacrée aux pays qui ont des revenus bas et aux femmes.

L'exemple d'un programme en Afrique de l'Est montre bien à quel point des investissements ciblés peuvent contribuer à faire reculer la pauvreté. Dans cette région, les petits producteurs laitiers connaissent une situation économique difficile, en partie à cause d'un accès déficient aux marchés et d'une dégradation des prairies. Le projet East Africa Dairy Development (EADD) est un programme de développement régional mis en œuvre au Kenya, en Ouganda et au Rwanda par I'ONG Heifer International et par un consortium de partenaires comprenant I'International Livestock Research Institute, le Centre international pour la recherche en agroforestrie, TechnoServe et Nestlé. Initié par la fondation Bill and Melinda Gates, le projet met en valeur les services et la formation afin d'améliorer les rendements agricoles et les revenus des petits agriculteurs. En outre, ce projet améliore I'accès aux marchés et réduit les pertes après récoltes. Vingt-sept centres de collecte sont développés pour le stockage et la réfrigération du lait. Ils sont gérés par de nouvelles associations commerciales. Quant aux petits producteurs, ils sont formés aux activités commerciales et à la production, avec un accent particulier mis sur des technologies de reproduction et des pratiques de nutrition animale améliorées pour une meilleure qualité du lait.

\section{Troisième recommandation : intensifier, de manière durable, la production agricole tout en réduisant les émissions de gaz à effet de serre issues de I'agriculture}

Une troisième recommandation consiste à intensifier, mais de manière durable, la production agricole tout en réduisant les émissions de gaz à effet de serre issues de l'agriculture.

Tout doit être fait pour favoriser le développement des systèmes agricoles bénéfiques qui permettent d'avoir des moyens de subsistance et des écosystèmes plus productifs et plus résilients. En particulier, il convient d'introduire des stratégies permettant de minimiser la dégradation des écosystèmes et de réhabiliter les environnements dégradés, en mettant l'accent sur les programmes conçus au niveau local.

Pour certaines cultures, cela suppose de mobiliser les producteurs marginalisés (en particulier les femmes) pour augmenter la productivité. Plus précisément, cette action passe par un renforcement des droits relatifs à l'eau et à la terre, par un meilleur accès aux marchés, aux financements et aux assurances et par le développement des capacités locales (par exemple via des organisations d'exploitants agricoles).

Par ailleurs, si des subventions incitent les exploitants à poursuivre des pratiques néfastes pour l'eau et les écosystèmes, elles devront être identifiées et modifiées. Plus largement, il convient de coupler les incitations économiques en faveur d'une intensification durable de l'agriculture avec une gouvernance renforcée des régimes fonciers et de l'affectation des terres. II s'agit ici de freiner le recul des forêts, des terres humides et des prairies.

Certains programmes en Chine montrent que l'augmentation de la productivité accompagne des efforts significatifs en matière de recherche et développement agricoles. Ainsi, le Plan pour la construction de projets de cultures protectrices doit couvrir 2,7 millions d'hectares entre 2009 et 2015. Outre I'amélioration de la résilience des sols à la sécheresse, entre 1,7 et 2,5 millions de mètres cubes d'eau d'irrigation ont été économisés. Des stratégies visant à améliorer les rendements rizicoles tout en réduisant les émissions de gaz à effet de serre ont été mises en avant. Ces stratégies encouragent notamment les agriculteurs à privilégier les variétés de riz à haut rendement, tout en ayant recours à des méthodes d'irrigation intermittente et en transformant la paille en matière première pour la production de combustible, de matériaux de construction et d'énergie. Des subventions ont été instaurées pour l'utilisation de tech- nologies, de machines et d'équipements $d$ 'irrigation économes en eau, ainsi que pour l'amélioration des systèmes industriels. Parmi toutes ces stratégies, celles qui auront été couronnées de succès seront intégrées dans les plans nationaux futurs. Les objectifs nationaux relatifs au changement climatique pour 2010, tels que $15 \%$ de ressources de combustible non fossile pour la consommation nationale d'énergie et 40 millions d'hectares de couverture forestière, ont été encouragés par des subventions, des labels et des incitations fiscales.

\section{Quatrième}

\section{recommandation : des programmes et des politiques spécifiques pour les populations et les secteurs les plus vulnérables face au changement climatique et à I'insécurité alimentaire}

La quatrième recommandation concerne les populations et les secteurs qui sont les plus vulnérables face au changement climatique et à l'insécurité alimentaire. Pour eux, il convient de mettre en œuvre des programmes et des politiques spécifiques.

Appliquer cette recommandation suppose d'abord de développer des financements ad hoc : citons ici les " fonds sur indices" (index-linked funds) qui apportent une aide rapide lorsque des évènements climatiques extrêmes touchent certaines communautés.

Il est par ailleurs impératif de modérer les fluctuations excessives des prix des produits alimentaires. La lutte contre la volatilité des prix implique d'abord de partager les informations nationales sur les prévisions de production et les stocks, en renforçant les bases de données sur les marchés, en mettant en avant des systèmes commerciaux ouverts et réceptifs, en établissant des systèmes d'avertissement précoces et en autorisant des exportations et des importations exemptes de taxes pour I'aide humanitaire.

Il convient également de créer et de maintenir des filets de sécurité pour aider les populations vulnérables à 
atteindre la sécurité alimentaire (par exemple, transferts de liquidités et en nature, programmes garantissant l'emploi, actions pour développer la résilience, santé et nutrition, éducation et cultures poussant rapidement en périodes de famine).

Enfin, il est important de créer et soutenir des plateformes destinées à coordonner les actions des donateurs internationaux, afin de veiller à intégrer systématiquement la gestion des risques liés au changement climatique, les avantages de l'adaptation et de l'atténuation et I'amélioration des résultats nutritionnels locaux.

Plus concrètement, II faut établir des réserves alimentaires d'urgence ainsi que des capacités de financement susceptibles d'apporter rapidement des réponses humanitaires aux populations menacées par les crises alimentaires.

En Éthiopie, l'approche orientée vers le développement à l'aide alimentaire adoptée par le Productive Safety Net Program (PSNP) se traduit par la création d'un filet de protection gouvernemental et une plus grande prévisibilité pour les petits paysans. En combinant les fonds des donateurs internationaux (plus de 1,27 milliard de dollars US au cours des six dernières années) avec l'infrastructure, la main d'œuvre et les intrants fournis par le gouvernement (500 000 USD par an), le PSNP assure un accès à la nourriture, stimule les marchés et réhabilite les ressources naturelles. Les familles victimes chroniques de l'insécurité alimentaire et qui comptent des adultes valides reçoivent de l'argent et de la nourriture pour leur participation aux travaux publics à forte quantité de main-d'œuvre, tandis que les autres familles reçoivent des virements inconditionnels. Ces initiatives de travaux publics visent à la qualité du sol, les ressources en eau, les conditions écologiques, l'infrastructure et les services sociaux. Actuellement dans sa troisième phase et opérationnel dans 317 woredas (divisions administratives), le programme PSNP atteint 7,7 millions de bénéficiaires. Ce programme connaît de nombreux succès : il a notamment fait sortir environ 1,3 million d'individus de I'insécurité alimentaire, il a permis de réhabiliter 9 millions $d^{\prime}$ 'hectares de terres tout en augmentant les cheptels, il a permis un meilleur accès à l'eau potable et suscité un développement de la scolarité.

\section{Cinquième \\ recommandation : repenser les modèles de consommation alimentaire pour s'assurer que les besoins nutritionnels de base sont garantis, tout en encourageant des modèles sains et durables}

La cinquième recommandation vise à repenser les modèles de consommation alimentaire pour s'assurer que les besoins nutritionnels de base sont garantis, tout en encourageant des modèles sains et durables à travers le monde.

Au titre de cette proposition, il convient en premier lieu de traiter la sousnutrition chronique et la famine : cet impératif suppose d'harmoniser les politiques de développement et de coordonner les programmes régionaux destinés à améliorer l'accès aux aliments pour toutes les populations qui vivent dans I'insécurité alimentaire.

Il importe également de promouvoir les changements positifs dans la variété et la quantité de régimes alimentaires : un tel changement passe par des campagnes pédagogiques innovantes, qui ciblent en particulier les jeunes consommateurs, ainsi que par des incitations économiques pour aligner les pratiques de marketing des revendeurs et des transformateurs sur les objectifs de santé publique et d'environnement.

Dans le même ordre d'idées, il convient de promouvoir un ensemble cohérent de normes afin de contrôler et d'évaluer la sécurité alimentaire, la nutrition et la santé, les pratiques de la chaîne d'approvisionnement, ainsi que les coûts et les avantages pour le système alimentaire. Ainsi, les consommateurs doivent avoir accès à un étiquetage clair et précis.

\section{Sixième \\ recommandation : réduire les pertes et gaspillages}

Dans sa sixième recommandation, la Commission met l'accent sur la nécessité de réduire les pertes et les gaspillages, en ciblant les infrastructures, les pratiques d'élevage, la transformation, la distribution et les habitudes des ménages.

À ce titre, il est intéressant d'observer que tous les programmes visant à développer une agriculture durable comportent un volet consacré à la réduction des gaspillages, et ce, depuis la production jusqu'à la consommation : il s'agit le plus souvent d'améliorer la gestion, pendant et après la récolte, ainsi que le stockage et le transport.

Citons ici l'innovation économique pour amener les producteurs à faibles revenus à stocker des produits pendant des périodes où l'offre est surabondante ou I'obligation pour les distributeurs de trier et de réduire le gaspillage alimentaire.

Les efforts entrepris pour limiter les pertes et gaspillages peuvent mobiliser des partenariats public-privé. Une initiative britannique, le Waste Resources and Action Programme (WRAP) fait appel aussi bien à des entreprises, des individus et des communautés pour réduire le gaspillage de nourriture, qui représente chaque année pas moins de 12 milliards de livres sterling de pertes et une émission de 20 millions de tonnes d'équivalent $\mathrm{CO}_{2}$. Les recherches du programme WRAP sont axées sur les moyens de réduire la quantité de nourriture jetée par les consommateurs et porte sur les habitudes, attitudes et comportements des consommateurs, sur les moyens appropriés de communiquer à des publics prioritaires et sur l'innovation dans le commerce de détail. En partenariat avec le WRAP, le secteur de l'épicerie s'est adapté pour permettre au consommateur d'acheter plus facilement uniquement la quantité de nourriture dont il a besoin et pour optimiser la fraîcheur des produits, mais aussi pour mettre en oeuvre des campagnes à grande échelle à l'intention des consommateurs (par exemple "Love Food Hate Waste"). 670000 tonnes de nourriture ont ainsi évité de se retrouver dans les décharges, soit une économie annuelle de 600 millions de livres (près de 750 millions d'euros).

\section{Septième recommandation : créer des systèmes d'informations complets, partagés et intégrés sur les denrées alimentaires}

La septième et dernière recommandation souligne la nécessité de créer des 
systèmes d'informations complets, partagés et intégrés sur les denrées alimentaires.

Des contrôles réguliers doivent pouvoir être menés, sur le terrain et par l'intermédiaire de réseaux de télédétection publics, afin de suivre les changements dans I'utilisation des terres, mais aussi la production alimentaire, le climat, I'environnement, la santé et le bien-être à l'échelle mondiale. II importe de d'accroître la transparence et l'accès aux informations relatives aux marchés alimentaires internationaux en investissant dans des systèmes d'informations interconnectés.

Plus particulièrement, que ce soit sur les précipitations, la température, I'humidité ou la sécheresse, il est essentiel pour les exploitants de disposer d'informations en temps réel et de prévisions fiables afin d'atténuer les effets du changement climatique et de s'adapter à ce dernier.

Ainsi, en Australie, ces défis doivent être relevés dans les conditions climatiques les plus instables de tous les continents habités. L'agriculture australienne représente au moins $25 \%$ des émissions de gaz à effet de serre du pays (soit à peu près la moyenne mondiale), défrichage compris, ce qui la contraint à la fois de réduire ses émissions de GES de 50 à $80 \%$ d'ici 2050 et d'augmenter sa production alimentaire de 30 à $80 \%$. La "Carbon Farming Initiative » australienne est la première législation nationale au monde d'atténuation du changement climatique lié aux terres et d'amélioration du revenu rural. Pour soutenir l'agriculture adaptative et le " carbon farming " des producteurs australiens, le bureau de météorologie (BoM) émet des perspectives climatiques saisonnières qui s'appuient sur des prévisions détaillées et des évaluations du risque, notamment une prévision à 3 mois et une prévision à 6 mois, basée sur l'oscillation australe El Niño. La Commonwealth Scientific and Industrial Research Organisation (CSIRO) et le BoM produisent également des projections climatiques à plus long terme pour aider les paysans à se préparer aux changements futurs. Dans certaines régions, comme dans le Sud-Ouest australien, les projections climatiques constituent déjà un outil important pour des exploitants obligés de modifier leurs pratiques agricoles en réaction au changement des conditions climatiques. Ces initiati- ves sont soutenues par le programme australien Farming Future qui fournit des informations, des financements, des formations et de l'assistance pour aider les paysans à s'adapter au changement climatique.

Il est intéressant de noter que les travaux et recommandations de la Commission ont été présentés et commentés depuis leur production lors de la rencontre de Durban sur le changement climatique, comme à Rio. Leur caractère non partisan et appuyé sur des analyses scientifiques partagées a sans doute joué un grand rôle dans l'intérêt qu'ils ont suscité dans ces enceintes.

Toutes les informations sur la Commission, son rapport final et ses préconisations à l'attention des décideurs politiques peuvent être consultées à l'adresse suivante : http://ccafs.cgiar. org/commission.

\section{Précisions}

La Commission internationale sur l'Agriculture durable et le Changement climatique est composée de :

- Professeur Sir John Beddington, Président, Royaume-Uni

- Dr Mohammed Asaduzzaman, Bangladesh Institute of Development Studies, Bangladesh

- Dr Adrian Fernández, Metropolitan University, Mexique

- Dr Megan Clark, Commonwealth Scientific and Industrial Research Organisation (CSIRO), Australie

- Dr Marion Guillou, Institut National de la Recherche Agronomique (INRA), France

- Professeur Molly Jahn, Université de Madison-Wisconsin, États-Unis

- Professeur Lin Erda, Académie chinoise des Sciences agricoles, Chine

- Professeur Tekalign Mamo, Ministère de I'Agriculture, Éthiopie

- Dr Nguyen Van Bo, Académie vietnamienne des sciences agricoles, Vietnam - Dr Carlos A Nobre, Ministère de la Science, de la Technologie et de I'Innovation, Brésil

- Professeur Robert Scholes, Council for Scientific and Industrial Research (CSIR), Afrique du Sud

- Dr Rita Sharma, National Advisory Council, Inde

- Professeur Judi Wakhungu, African Center for Technology Studies (ACTS), Kenya.
Conflits d'intérêts : aucun

\section{RÉFÉRENCES}

Bai ZG, Dent DL, Olsson L, Schaepman ME. Global assessment of land degradation and improvement identification by remote sensing. Wageningen: International Soil Reference and Information Centre (ISRIC). 2008.

Bruinsma J. The resource outlook to 2050: by how much do land, water and crop yields need to increase by 2050? Expert Meeting on How to Feed the World in 2050. Rome, FAO, 2009.

FAO. The state of food insecurity in the world: addressing food insecurity in protracted crises, 2010. www.fao.org/docrep/013/ i1683e/i1683e.pdf.

Foley, et al. Solutions for a cultivated planet. Nature 2011 ; 478 : 337-42.

Foresight. Tackling Obesities: Future Choices. Project Report. London: UK: Government Office for Science. Department for Business, Innovation and Skills, 2007.

Gustavsson, et al. Global food losses and food waste. Rome, Italy: Food and Agriculture Organization of the United Nations, 2011. www.fao.org/fileadmin/user_upload/ags/ publications/GFL_web.pdf.

Haslam DW, James WPT. Obesity. Lancet $2005 ; 366: 1197-209$.

Munich RE Geo Risks Research NatCatSERVICE. Natural catastrophes worldwide, 2010. http://www.munichre.com/en/reinsurance/ business/nonlife/georisks/natcatservice/ annual_statistics.aspx.

United Nations Convention to Combat Desertification. Desertification: A visual synthesis. Bonn, Germany: UNCCD Secretariat. www. unccd.int/knowledge/docs/Desertification-EN. pdf2011.

United Nations Population Division. World population prospects: the 2010 revision. New York : United Nations Department of Economic and Social Affairs United Nations Population Division, 2010. http://esa.un. org/wpp/unpp/panel_population.htm.

World Health Organization. Obesity and overweight. Fact sheet No. 311, 2011. www.who.int/mediacentre/factsheets/fs311/ en/.

WHO/FAO. Diet, Nutrition and the Prevention of Chronic Diseases. Genève : WHO, 2003.

World Bank. The Hague Conference on Agriculture, Food Security and Climate Change Opportunities and Challenges for a Converging Agenda: Country Examples. Conference Edition, 2010a. 


\section{POUR EN SAVOIR PLUS}

Africare, Oxfam America, WWF-ICRISAT Project.. More Rice for People: More Water for the Planet, System of Rice Intensification (SRI). Hyderabad, Inde: WWF-ICRISAT Project, 2010.

Barrett CB, Barnett B], Carter MR, Chantarat S, Hansen JW, Mude AG, Osgood D, Skees JR, Turvey CG, Ward MN. Poverty traps and climate risk: limitations and opportunities of index-based risk financing. IRI Technical Report No. 07-02. Columbia University: International Research Institute for Climate and Society, 2007.

Cabinet Office. Food Matters: A strategy for the 21st century. Londres: Cabinet Office, 2008.

Chen KZ, Zhang T. Foresight Project on Global Food and Farming Futures. Regional case study R2: agricultural $R \& D$ as an engine of productivity growth: Chine. 2011www. bis.gov.uk/assets/bispartners/foresight/docs/ food-andfarming/ regional/11-591-r2-agricultural-r-and-d-productivity-growth-china. pdf. Conversion at interbank rates of 15 June 2007 at www.oanda.com.

Choudhury ML. Recent developments in reducing post-harvest losses in the AsiaPacific region. In: R. S. Rolle, ed., Reports of the APO Seminar on Reduction of Postharvest Losses of Fruit and Vegetables, October 2004 : 5-11. Tokyo, Japon : FAO, Asian Productivity Organization (APO), 2006.

Cline WR. Global Warming and Agriculture: Impact Estimates by Country. Washington DC, USA. Peterson Institute, 2007. http://maps. grida.no/go/graphic/projected-agriculture-in2080-due-to-climatechange

Etilé F. Food Consumption and Health. In: J. Lusk, J. Roosen, J. Shogren (Eds.), Oxford Handbook of the Economics of Food and Agricultural Policy. Oxford, UK : Oxford University Press, 2010.

FAO. The State of the Food Insecurity in the World: How does international price volatility affect domestic economies and food security? Rome, Italie: Food and Agriculture Organization of the United Nations. 2011a.

FAO. State of the World's Forests. Rome, Italie : Food and Agriculture Organization of the United Nations. 2011b.

FAO. The State of Agricultural Commodity Markets. High food prices and the foodcrisis experiences and lessons learned. Food and Agriculture Organization of the United Nations. Rome, Mars 2009.

FAO Country Profiles. China: Food Safety and Security. http://www.fao.org/countryprofiles/ index.asp?lang=en\&iso3=CHN\&paia $=2$

FAO Country Profiles. France: Food Safety and Security. http://www.fao.org/fileadmin/ templates/ess/documents/food_security_ statistics/country_profiles/eng/France_E.pdf

FAO Country Profiles. South Africa: Food Safety and Security. http://www.fao.org/ countryprofiles/index.asp?lang=en\&iso3= ZAF\& paia $=2$

FAO Stat. Mexico. http://faostat.fao.org/site/ 666/default.aspx

Foresight. The Future of Food and Farming. Final Project Report. Futures. Londres, GB : Government Office for Science. 2011

Foresight. Migration and Global Environmental Change: Future Challenges and Opportunities. 2011. Final Project Report. Futures. Londres, GB : Government Office for Science.

Fuchs A, Wolff H. Concept and unintended consequences of weather index insurance: the case of Mexico. American Journal of Agricultural Economics 2011 ; 93 : 505-11.

Government of the People's Republic of Bangladesh. Bangladesh Country Investment Plan: a road map towards investment in agriculture, food security and nutrition. Updated Version June 2011. 2011www. nfpcsp.org/agridrupal/sites/default/files/ Bangladesh\%20Food\%20security\%20CIP\% 202011\%20Final.pdf

Hazell PBR, Pomerada C, Valdes A (Eds.). Crop Insurance for agricultural development: issues and experience. Washington DC : International Food Policy Research Institute, 1986.

Hess U, Hazell P. Innovations in insuring the poor. Sustainability and scalability of indexbased insurance for agriculture and rural livelihoods. 2020 Vision. Focus 17. Brief 5. Washington DC : International Food Policy Research Institute, 2009.

HMG. The 2007/2008 Agricultural Price Spikes: Causes and Policy Implications. Londres : HM Government, 2010.

IFAD. Rural Poverty Report: New realities, new challenges: new opportunities for tomorrow's generation. Rome, Italie : International Fund for Agricultural Development, 2011.

IFPRI. Gilligan DO, Hoddinott J, Kumar NR, Taffesse AS. An Impact Evaluation of Ethiopia's Productive Safety Nets Program. International Food Policy Research Institute, 2033 K Street, NW, Washington, D.C. 20006, 2008.

INRA/CIRAD. Agrimonde: Scenarios and Challenges for Feeding the World in 2050. Versailles, France : Editions Quae, 2011.

International Assessment of Agricultural Knowledge, Science and Technology for Development (IAASTD). Agriculture at a crossroads: A synthesis of the global and sub-global IAASTD reports, eds. B.D. Mclntyre, H.R. Herren, J. Wakhungu, and R.T.
Watson. Washington, D : Island Press, 2009.

Jolly R. Junk food, advertising and kids. Research Paper No. 9 2010-11. Parliament of Australia Parliamentary Library. 2011. www.aph.gov.au/library/pubs/rp/2010-11/ 11 rp09.htm\#_Toc282609530

Kachika T. Women's land rights in Southern Africa. Afrique du Sud : Niza and ActionAid International. 2009www.niza.nl/documenten/100129_womens_land_rights.pdf

Laborte AG, de Bie K, Smaling EMA, Moya PF, Boling AA, Van Ittersum MK. Rice yields and yield gaps in Southeast Asia: Past trends and future outlook. Europ / Agronomy 2011; 36 (2012) : 9.

Lipper L, Mann W, Meybeck A, Sessa R. "Climate-Smart" Agriculture: Policies, Practices and Financing for Food Security, Adaptation and Mitigation. Rome, Italie : UN Food and Agriculture Organization, 2010.

Mahapatra R.. MGNREGA: making way for women's empowerment. 2010. www.southasia. oneworld.net/fromthegrassroots/mgnregapaving-way-for-womenempowerment

Meridian. Addressing agriculture in climate change negotiations: a scoping report. Colorado and Washington DC: Meridian Institute, 2011.

Millennium Ecosystem Assessment. Ecosystems and Human Well-being. 2005.

Millennium Ecosystem Assessment. Ecosystems and Human Well-being: Synthesis. Washington, DC : Island Press, 2005.

Mittal S. Strengthening backward and forward linkages in horticulture: some successful initiatives. Agric Econ Res Rev 2007 ; 20 : 457-69.

National Academy of Sciences. Toward Sustainable Agricultural Systems in the 21 st Century. Washington, DC : The National Academies Press, 2010.

National Development and Reform Commission. China's policies and actions for addressing climate change -The Progress Report 2009. China: National Development and Reform Commission, 2009. www.ccchina. gov.cn/WebSite/CCChina/UpFile/File571.pdf Nelson GC, Rosegrant MW, Palazzo A, et al. Climate change: Impact on Agriculture and Costs of Adaptation and Food Security, Farming, and Climate Change to 2050. Washington, DC : International Food Policy Research Institute, 2011.

Nelson GC, Rosegrant MW, Koo J, et al. Climate change: impact on agriculture and costs of adaptation. Washington DC : International Food Policy Research Institute, 2009.

Nguyen VT, Nguyen QT, Nguyen VA. Influence of on farm water management to the methane emissions in the Red River Delta 
Area - Vietnam. Vietnam National Commission on Large Dams, 2007.

Parry M, Evans A, Rosegrant MW, Wheeler T. Climate Change and Hunger: Responding to the Challenge. Rome, Italie: World Food Program, 2009.

Prosser IP (Ed.). Water: science and solutions for Australia. Australie: CSIRO Publishing, 2011.

Reardon $\mathrm{T}$, Timmer $\mathrm{CP}$, Barrett $\mathrm{CB}$, Berdegué J. The Rise in Supermarkets in Africa, Asia, and Latin America. American Journal of Agricultural Economics 2003 ; 85 : 1140-6.

Royal Society. Reaping the Benefits: Science and the Sustainable Intensification of Global Agriculture. Londres: The Royal Society, 2009.

The Hague Conference on Agriculture, Food Security and Climate Change. Chair's Summary, 2010.
USDA.. What does the "Organic" label really mean? US Department of Agriculture (USDA) and the Food and Drug Administration (FDA). 2011; http://usdafda. com/articles/ organic.htm

Vermeulen SJ, Aggarwal PK, Ainslie A, et al. Agriculture, Food Security and Climate Change: Outlook for Knowledge, Tools and Action. CCAFS Report 3. Copenhagen, Denmark: CGIAR-ESSP Program on Climate Change, Agriculture and Food Security, 2010.

World Bank. Climate-smart agriculture: increased productivity and food security, enhancing resilience and reduced carbon emissions for sustainable development, opportunities and challenges for a converging agenda: country examples. Washington DC : The World Bank, 2011a.

World Bank. Food Price Watch. 2011b. http://siteresources.worldbank.org/INTPO-
VERTY/Resources/335642- 1210859591030/ FPW_April2011.pdf

World Bank. World Development Report, 2010: Development and Climate Change. Washington, DC : The World Bank, $2010 \mathrm{~b}$.

World Bank. Designing and implementing a rural safety net in a low income setting: Lessons learned from Ethiopia's Productive Safety Net Program 2005- 2009. Independent Evaluation Group of the World Bank's assistance to social safety net, 2010c.

World Bank. World Development Report, 2008: Development and Agriculture. Washington, DC : The World Bank, 2008.

Worldwatch Institute. State of the World: Innovations that Nourish the Planet. New York, NY USA : W.W. Norton \& Company, 2011.

WRAP. The Food We Waste, Banbury. Waste and Resources Action Programme, 2008. 